\title{
Diferencias según el género en el perfil clínico de riesgo del paciente amputado de miembro inferior
}

\author{
Gender differences within the clinical risk profile of lower-limb amputee patients
}

\author{
María Gutiérrez-Fernández ${ }^{1,2 *}$, David Carrasco-De Andrés³, Luis M. Salmerón-Febres ${ }^{4}$ \\ Lucas González-Herrera ${ }^{4}$ y Silvia Jiménez-Brobeil ${ }^{1}$ \\ 'Departamento de Medicina Legal y Forense, Toxicología y Antropología Física y Forense, Facultad de Medicina de Granada, Universidad de

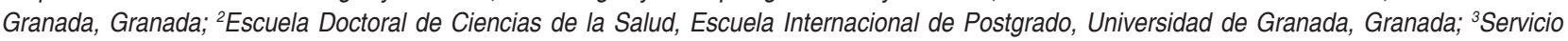 \\ de Angiología y Cirugía Vascular, Hospital HLA Mediterráneo, Almería; ${ }^{4}$ Servicio de Angiología y Cirugía Vascular, Hospital Universitario San \\ Cecilio, Granada. España
}

\section{Resumen}

Objetivo: Analizar las diferencias según el sexo en el perfil clínico de riesgo de los pacientes amputados de miembro inferior por causa no traumática. Método: Estudio retrospectivo descriptivo con 697 pacientes amputados de miembro inferior por causa no traumática, en un Servicio de Angiología y Cirugía Vascular, durante un periodo de 5 años y divididos en función del sexo. Se analizaron variables demográficas (edad) y clínicas (causa, comorbilidad, factores de riesgo cardiovascular y nivel de amputación). Resultados: La edad media ( \pm desviación estándar) de las mujeres fue mayor (75.3 \pm 12 frente a 68.9 \pm 11 años; $p$ < 0.001) y ellas presentaron mayor frecuencia de factores de riesgo cardiovascular concomitantes, tales como diabetes, hipertensión y obesidad, que los varones, mientras que ellos presentaron mayor frecuencia de tabaquismo. No hubo diferencia significativa entre sexos respecto a la distribución de las causas de amputación, siendo la principal causa la isquémica, destacando la arteriosclerosis. Las mujeres presentaron mayor frecuencia y predisposición para un nivel de amputación mayor. Conclusiones: En nuestro estudio, las mujeres presentaron un perfil de riesgo cardiovascular más desfavorable y el sexo femenino fue un predictor independiente de amputación mayor de miembro inferior por causa no traumática.

Palabras clave: Amputación. Extremidad inferior. Sexo. Factores de riesgo cardiovascular.

\begin{abstract}
Objectives: To analyse the possible gender differences within the clinical risk profile of lower-limb amputee patients who had a non-traumatic amputation. Method: A retrospective study with 697 lower-limb amputee patients, classified according to their gender, has been conducted at the Angiology and Vascular Surgery Department for 5 years. The variables considered were demographic variables (age and gender) and clinic variables (cause of the amputation, comorbidity, cardiovascular risk factors and the amputation level). Results: Regarding to the results, the average of women was older than men (75.3 $\pm 12 \mathrm{vs.} 68.9$ \pm 11 years; $p<0.001$ ), showing an increasing frequency to concomitant cardiovascular risk factors, such as diabetes, hypertension, and obesity. However, men present a higher frequency to smoking. There were no gender differences in the main
\end{abstract}

\section{Correspondencia:}

*María Gutiérrez-Fernández

Avda. de la Investigación 11

C.P. 18016, Granada, España

Fecha de recepción: 15-06-2020

E-mail: gutierrez_peritacion@ hotmail.es;

gutierrezperitacion@correo.ugr.es
Cir Cir. 2021;89(4):490-496

Contents available at PubMed www.cirugiaycirujanos.com ajo la licencia CC BY-NC-ND (http://creativecommons.org/licenses/by-nc-nd/4.0/) 
cause of the amputation, the ischemic cause, being notably into this cause the subgroup of arteriosclerosis cause. Women showed a higher frequency and predisposition for major amputation. Conclusions: This study showed that women had a more unfavorable cardiovascular risk profile than men, being the female gender an independent predictor to a major lower-limb amputation with non-traumatic amputation.

Key words: Amputation. Lower limb. Gender. Cardiovascular risk factors.

\section{Introducción}

En el panorama internacional actual, España ocupa el segundo lugar en cuanto a la tasa de amputación de miembro inferior (MI) por pie diabético, con evidente diferencia en función del sexo (4.71 en hombres frente a 1.75 en mujeres) y únicamente superado por los Estados Unidos de América, con una tasa de amputación de 3.19 por cada 1000 personas ${ }^{1,2}$. Se trata de un problema de salud en auge, puesto que mientras la tasa de amputaciones de MI de origen traumático o neoplásico disminuye considerablemente con el tiempo, es un hecho el progresivo incremento de la tasa de amputación de MI por causa no traumática, a pesar de los grandes avances en el campo de las ciencias quirúrgicas.

Analizando los datos demográficos de algunos estudios sobre este tipo de cirugía de las últimas décadas $^{3-5}$, se debe considerar la edad como un factor determinante, ya que se trata fundamentalmente de pacientes de edad avanzada, con mayor incidencia de enfermedad vascular periférica, de factores de riesgo cardiovascular (FRCV) y patología concomitante. El aumento de la esperanza de vida en España, junto con los trastornos vasculares de la tercera edad, favorecen sin duda el aumento de estos eventos, siendo la edad un factor fundamental en la amputación no traumática de MI.

También en estas últimas investigaciones parece que el sexo del paciente puede ser un factor diferencial, ya que se evidencia una clara desigualdad de amputaciones de Ml entre sexos. En la actualidad, los grandes afectados por este tipo de eventos son los varones, en una proporción 3 a 1 aproximadamente ${ }^{6,7}$. Aunque no se ha demostrado que el sexo sea un factor de riesgo para sufrir un acto quirúrgico de este tipo, es posible que exista un perfil clínico específico en función de un determinado sexo, que explique una mayor tendencia a la amputación de Ml en los varones.

La mayor parte de la evidencia científica en la que se basa la actuación médica actual en los pacientes amputados de Ml se ha obtenido de estudios con una mayor participación de hombres. Sin embargo, no debemos obviar que, a pesar de que los varones se vean más afectados por estos actos quirúrgicos, tanto las enfermedades vasculares en general como las amputaciones de MI por causa vascular en particular constituyen una importante y creciente fuente de morbimortalidad en la mujer.

Hasta hace pocos años, tanto los registros promovidos por instituciones científicas como los ensayos clínicos de intervención han incluido una mínima proporción de mujeres, generando un gran vacío científico en el conocimiento de este tipo de eventos en el sexo femenino.

En este sentido, y ante la falta de publicaciones acerca de este creciente problema vascular que traten con la misma profundidad ambos sexos, a pesar de las evidentes desigualdades entre hombres y mujeres que se desprenden de los datos de las investigaciones existentes, así como de la propia evidencia clínica, este estudio tiene como propósito principal examinar las posibles diferencias en el perfil clínico del paciente amputado de MI por causa no traumática de forma bifocal, en función del sexo, aportando mayor conocimiento a este complejo dilema de salud.

\section{Método}

\section{Diseño del estudio}

Se realizó un estudio clínico retrospectivo descriptivo con 697 pacientes amputados de Ml en el Servicio de Angiología y Cirugía Vascular del Hospital Universitario San Cecilio de Granada, España, durante un periodo de 5 años, comprendido entre el 1 de enero de 2009 y el 1 de enero de 2014.

\section{Muestra}

Los pacientes se dividieron en dos grupos en función del sexo, y los criterios de inclusión fueron ser mayor de edad, sufrir amputación de $\mathrm{Ml}$ en el centro hospitalario durante el periodo de estudio y amputación de causa no traumática. 
Los pacientes amputados en más de una ocasión en el periodo de estudio se registraron por el mayor nivel de amputación, para evitar duplicidades.

\section{Variables estudiadas}

Se analizaron dos tipos de variables: demográficas (edad y sexo) y clínicas (FRCV, causa, comorbilidad y nivel de amputación).

Los FRCV estudiados fueron diabetes, hipertensión, dislipidemia, tabaquismo y obesidad. Se recogieron de la historia clínica de los pacientes, utilizando como criterio el diagnóstico previo de dichos factores registrado en la historia clínica. El tabaquismo se definió como el hábito crónico de fumar. La obesidad se definió con un índice de masa corporal $\geq 30 \mathrm{~kg} / \mathrm{m}^{2}$.

Los pacientes se clasificaron en dos grupos atendiendo a la causa: isquémica y no isquémica. Dentro de la causa isquémica se distinguió entre causa arteriosclerótica (isquemia crónica de MI) y causa tromboembólica (isquemia aguda de MI). En la causa no isquémica se incluye el pie diabético neuropático y séptico.

La comorbilidad considerada incluyó cardiopatía isquémica, arritmia cardiaca, insuficiencia cardiaca, insuficiencia renal y retinopatía diabética. Se utilizó como criterio el diagnóstico previo de dichas patologías registrado en la historia clínica.

Se definieron dos categorías de nivel de amputación, de acuerdo con la clasificación de la Sociedad Andaluza de Angiología y Cirugía Vascular: amputación menor (conservando el talón) y amputación mayor.

\section{Análisis estadístico}

Se utilizó el programa SPSS versión 25.0. Se realizó una comparación de todas las características demográficas y clínicas de los pacientes en función del sexo. Las variables categóricas se compararon mediante la prueba de ji al cuadrado, mientras que las variables continuas se compararon con la prueba ANOVA. El umbral de significación se estableció en $\alpha=0.05$.

Se llevó a cabo, además, un modelo de regresión logística multivariante para conocer los factores asociados a la amputación mayor. En el modelo de regresión logística se incluyeron la edad, el sexo y las variables clínicas que podían estar en relación con la necesidad de sufrir una amputación mayor. Se tomaron como variables independientes los principales factores pronóstico para una amputación mayor: edad, sexo, FCRV y comorbilidad.

\section{Resultados}

Las características demográficas y la distribución de los FRCV de los pacientes amputados de MI, en función del sexo, se resumen en la tabla 1 . Se incluyeron 697 pacientes, con una edad media de 70.5 años (DE: 11.9), siendo el mínimo de 27 años y el máximo de 98 años, y una mediana de 72 años. El número de hombres amputados triplicó al de mujeres. El sexo femenino presentó una edad media significativamente mayor que el masculino y un mayor porcentaje en el subgrupo de edad de más de 65 años.

La diabetes, la hipertensión y la obesidad fueron los FRCV que se presentaron con mayor frecuencia y con una relación estadísticamente significativa en las mujeres, mientras que el tabaquismo predomino de forma significativa en los hombres.

En cuanto a la comorbilidad, no hubo diferencias estadísticamente significativas entre ambos sexos, tal como se muestra en la tabla 2. También se analizó la frecuencia de amputación mayor, en la que se observó una mayor proporción en el sexo femenino, con una diferencia estadísticamente significativa.

La tabla 3 resume el análisis de las causas de amputación, entre las que no se encontró diferencia entre hombres y mujeres, respecto a las dos causas generales (isquémica y no isquémica), destacando en ambos sexos dentro de la causa isquémica la arteriosclerosis.

En la tabla 4 se muestran los resultados del estudio de regresión logística multivariante realizado. Las variables que favorecen la posibilidad de sufrir una amputación mayor son la edad, el sexo femenino, la diabetes, la patología cardiaca previa y la retinopatía diabética, dado que fueron predictores independientes de amputación mayor.

\section{Discusión}

Los resultados de este estudio clínico descriptivo realizado sobre una amplia muestra de pacientes amputados de MI por causa no traumática muestran la relevancia del sexo en estos actos quirúrgicos. El trabajo, llevado a cabo en un servicio de angiología y cirugía vascular de un hospital público, es representativo de cómo el sexo femenino condiciona un perfil clínico más desfavorable. 
Tabla 1. Características demográficas y factores de riesgo cardiovascular según el sexo

\begin{tabular}{|c|c|c|c|c|}
\hline & $\begin{array}{c}\text { Total } \\
\text { pacientes } \\
(\mathrm{n}=697)\end{array}$ & $\begin{array}{c}\text { Hombres } \\
74.9 \%(n=522)\end{array}$ & $\begin{array}{c}\text { Mujeres } \\
25.1 \%(n=175)\end{array}$ & $p$ \\
\hline Edad media & $70.5 \pm 11.9$ & $68.9 \pm 11$ & $75.3 \pm 12$ & 0.001 \\
\hline $\begin{array}{l}\text { Mediana } \\
>65 \text { años }\end{array}$ & $\begin{array}{c}72 \\
70.4 \% \\
(n=491)\end{array}$ & $\begin{array}{c}69 \%(n=339) \\
H: 64.9\end{array}$ & $\begin{array}{c}31 \%(n=152) \\
M: 86.9 \%\end{array}$ & 0.020 \\
\hline \multicolumn{5}{|l|}{ FRCV } \\
\hline Diabetes & $\begin{array}{c}78.5 \% \\
(n=547)\end{array}$ & $\begin{array}{c}73.1 \%(n=400) \\
H: 76.6\end{array}$ & $\begin{array}{c}26.9 \%(n=147) \\
\text { M: } 84 \%\end{array}$ & 0.040 \\
\hline Hipertensión & $\begin{array}{c}58.8 \% \\
(n=410)\end{array}$ & $\begin{array}{c}71 \%(n=291) \\
H: 55.6 \%\end{array}$ & $\begin{array}{c}29 \%(n=119) \\
\text { M: } 68 \%\end{array}$ & 0.003 \\
\hline Dislipidemia & $\begin{array}{c}33.9 \% \\
(n=236)\end{array}$ & $\begin{array}{c}72.9 \%(n=172) \\
H: 32.8 \%\end{array}$ & $\begin{array}{c}27.1 \%(n=64) \\
\text { M: } 36.5 \%\end{array}$ & 0.140 \\
\hline Tabaquismo & $\begin{array}{c}50.8 \% \\
(n=354)\end{array}$ & $\begin{array}{c}96.3 \%(n=341) \\
H: 65.3 \%\end{array}$ & $\begin{array}{c}3.7 \%(n=13) \\
\text { M: } 7.4 \%\end{array}$ & 0.001 \\
\hline Obesidad & $\begin{array}{c}27 \% \\
(n=188)\end{array}$ & $\begin{array}{c}63.8 \%(n=120) \\
H: 23 \%\end{array}$ & $\begin{array}{c}6.2 \%(n=68) \\
\text { M: } 8.6 \%\end{array}$ & 0.001 \\
\hline
\end{tabular}

DE: desviación estándar; FRCV: factores de riesgo cardiovascular; H: hombres; M: mujeres.

El parámetro $n$ se ha redondeado en todos los casos a números enteros, ya que indica el número de individuos de la muestra afectados.

Tabla 2. Comorbilidad y amputación mayor según el sexo

\begin{tabular}{|c|c|c|c|c|}
\hline & $\begin{array}{c}\text { Total } \\
\text { pacientes } \\
(n=697)\end{array}$ & $\begin{array}{c}\text { Hombres } \\
74.9 \%(n=522)\end{array}$ & $\begin{array}{c}\text { Mujeres } \\
25.1 \%(n=175)\end{array}$ & $p$ \\
\hline $\begin{array}{l}\text { Amputación } \\
\text { mayor }\end{array}$ & $\begin{array}{c}54.7 \% \\
(n=381)\end{array}$ & $\begin{array}{c}71.4 \%(n=272) \\
H: 52.1 \%\end{array}$ & $\begin{array}{c}28.6 \%(n=109) \\
M: 62.3 \%\end{array}$ & 0.019 \\
\hline \multicolumn{5}{|l|}{ Comorbilidad } \\
\hline $\begin{array}{l}\text { Cardiopatía } \\
\text { isquémica } \\
\text { previa }\end{array}$ & $\begin{array}{l}12.1 \% \\
(n=84)\end{array}$ & $\begin{array}{c}76.2 \%(n=64) \\
H: 12.3 \%\end{array}$ & $\begin{array}{c}23.8 \%(n=20) \\
\text { M: } 11.4 \%\end{array}$ & 0.69 \\
\hline $\begin{array}{l}\text { Arritmia } \\
\text { cardíaca } \\
\text { previa }\end{array}$ & $\begin{array}{l}11.6 \% \\
(n=81)\end{array}$ & $\begin{array}{c}74.1 \%(n=60) \\
H: 11.5 \%\end{array}$ & $\begin{array}{c}25.9 \%(n=21) \\
\text { M: } 12 \%\end{array}$ & 0.69 \\
\hline $\begin{array}{l}\text { Insuficiencia } \\
\text { cardiaca } \\
\text { previa }\end{array}$ & $\begin{array}{c}6.2 \% \\
(n=43)\end{array}$ & $\begin{array}{c}67.4 \%(n=29) \\
\text { H: } 5.6 \%\end{array}$ & $\begin{array}{c}32.6 \%(n=14) \\
M: 8 \%\end{array}$ & 0.69 \\
\hline $\begin{array}{l}\text { Insuficiencia } \\
\text { renal previa }\end{array}$ & $\begin{array}{c}14.3 \% \\
(n=100)\end{array}$ & $\begin{array}{c}74 \% \mathrm{n}(=74) \\
\mathrm{H}: 14.2 \%\end{array}$ & $\begin{array}{c}26 \%(n=26) \\
\text { M: } 14.9 \%\end{array}$ & 0.82 \\
\hline $\begin{array}{l}\text { Retinopatía } \\
\text { diabética } \\
\text { previa }\end{array}$ & $\begin{array}{c}18.5 \% \\
(n=129)\end{array}$ & $\begin{array}{c}78.3 \%(n=101) \\
H: 19.4 \%\end{array}$ & $\begin{array}{c}21.7 \%(n=28) \\
M: 16 \%\end{array}$ & 0.71 \\
\hline
\end{tabular}

H: hombres; M: mujeres.

El parámetro $n$ se ha redondeado en todos los casos a números enteros, ya que indica el número de individuos de la muestra afectados.

Las mujeres amputadas presentaron un promedio de edad de 6.4 años más y mayor prevalencia de diabetes que los hombres, quienes únicamente las superaban en la tasa de tabaquismo. Estos hallazgos son similares a los presentados en el estudio de Davie-Smith $F$, et al. ${ }^{9}$ sobre el impacto del sexo en la
Tabla 3. Distribución de las causas de amputación según el sexo

\begin{tabular}{|c|c|c|c|c|}
\hline & $\begin{array}{c}\text { Total } \\
\text { pacientes } \\
(n=697)\end{array}$ & $\begin{array}{c}\text { Hombres } \\
\text { s74,9\% }(n=522)\end{array}$ & $\begin{array}{c}\text { Mujeres } \\
25,1 \%(n=175)\end{array}$ & $p$ \\
\hline Causa isquémica: & $\begin{array}{c}71,7 \% \\
(n=500)\end{array}$ & $\begin{array}{c}74 \%(n=370) \\
H: 70,9 \%\end{array}$ & $\begin{array}{c}26 \%(n=130) \\
M: 74,3 \%\end{array}$ & 0,62 \\
\hline $\begin{array}{l}\text { - Causa } \\
\text { arteriosclerótica }\end{array}$ & $\begin{array}{l}64,7 \% \\
(=451)\end{array}$ & $\begin{array}{c}73,8 \%(n=333) \\
H: 63,8 \%\end{array}$ & $\begin{array}{c}26,2 \%(n=118) \\
\text { M: } 67,4 \%\end{array}$ & 0,83 \\
\hline $\begin{array}{l}\text { - Causa } \\
\text { tromboembólica }\end{array}$ & $7 \%(49)$ & $\begin{array}{c}75,5 \%(n=37) \\
H: 7,1 \%\end{array}$ & $\begin{array}{c}24,5 \%(n=12) \\
M: 6,9 \%\end{array}$ & 0,83 \\
\hline Causa no isquémica & $\begin{array}{c}28,3 \% \\
(n=197)\end{array}$ & $\begin{array}{c}77,2 \%(n=152) \\
H: 29,1 \%\end{array}$ & $\begin{array}{c}22,8 \%(n=45) \\
\text { M: } 25,7 \%\end{array}$ & 0.62 \\
\hline
\end{tabular}

\section{H: hombres: M: mujeres.}

El parámetro $n$ se ha redondeado en todos los casos a números enteros, ya que indica

el número de individuos de la muestra afectados.

Tabla 4. Modelo de regresión logística paso a paso con carácter predictivo de la práctica de una amputación mayor de miembro inferior de la población de estudio

\begin{tabular}{lcc}
\hline Amputación mayor & $\mathbf{p}$ & OR (IC95\%) \\
\hline Edad & 0.008 & $0.953(0.947-0.967)$ \\
Sexo & 0.019 & $1.195(1.037-1.377)$ \\
FRCV & & \\
$\quad$ Diabetes mellitus & $<0.001$ & $1.889(1.691-2.109)$ \\
Hipertensión & 0.170 & $1.236(0.913-1.674)$ \\
Dislipidemia & 0.873 & $1.026(0.749-1.406)$ \\
Tabaquismo & 0.704 & $1.059(0.786-1.428)$ \\
Obesidad & 0.187 & $0.978(0.699-1.368)$ \\
& & $0.821(0.697-1.077)$ \\
Comorbilidad & & \\
$\quad$ Patología cardiaca previa & 0.002 & $1.674(1.200-2.336)$ \\
Insuficiencia renal previa & 0.886 & $0.969(0.634-1.486)$ \\
Retinopatía diabética & $<0.001$ & $1.503(1.197-1.888)$ \\
\hline
\end{tabular}

IC95\%: intervalo de confianza del 95\%; OR: odds ratio.

tasa de ajuste protésica tras una amputación mayor de $\mathrm{MI}$, realizado en Escocia.

De acuerdo con recientes estudios nacionales ${ }^{10-12} \mathrm{e}$ internacionales ${ }^{13-15}$, los resultados de este trabajo muestran que los grandes afectados por estas intervenciones son los hombres, cuyo porcentaje de amputados triplica al del mujeres, a pesar de que estas presentan una edad media más alta y peor perfil clínico previo.

Sin embargo, aunque la frecuencia de amputación es muy inferior en las mujeres, en ellas la proporción de amputación mayor observada superó significativamente a la de los hombres.

Desde el punto de vista fisiopatológico, los FRCV presentes en el paciente tienen un papel fundamental en el desenlace de este tipo de eventos quirúrgicos, ya que determinan en gran medida el nivel de amputación, la evolución y el pronóstico. 
El hecho de que más de tres cuartas partes de las amputaciones de los pacientes de la muestra de este estudio fueron motivadas por un problema vascular de tipo isquémico del $\mathrm{Ml}$ refuerza la importancia que tiene el perfil cardiovascular previo del paciente, lo que estaría en consonancia con los hallazgos de otras publicaciones ${ }^{16-18}$, en las que también se destaca la importancia de los FRCV sobre la amputación de MI.

Además de un peor perfil clínico en las mujeres amputadas, es posible que estas presenten características específicas del sexo que favorezcan un nivel superior de amputación que en los hombres, a pesar de la baja frecuencia de estas intervenciones en comparación con el sexo masculino.

Estos aspectos diferenciales asociados al sexo, evidentes en la práctica clínica habitual y que podrían favorecer tanto un peor perfil cardiovascular como un nivel superior de amputación en el sexo femenino, todavía no son bien conocidos, fundamentalmente por la insuficiente inclusión de mujeres en los ensayos clínicos y en otros estudios sobre este tipo de cirugía.

Analizando los distintos FRCV, en el caso de la diabetes existen estudios que recogen un incremento de la glucemia y de la incidencia de diabetes tipo 2 con la edad en ambos sexos, aunque con un claro predominio del sexo femenino ${ }^{19-21}$. Estas publicaciones muestran un predominio del sexo femenino en respuesta a la mayor presencia de obesidad con aumento de la resistencia a la insulina, la mala distribución de la grasa corporal, la pérdida de la función protectora de los estrógenos y una mayor expectativa de vida en las mujeres. Los resultados de este trabajo muestran diferencias significativas en la distribución de este FRCV entre los pacientes amputados en función del sexo, con mayor frecuencia de diabetes en las mujeres, de acuerdo con dichas publicaciones. Según Peters, et al. ${ }^{22}$, un mayor deterioro en el perfil de riesgo cardiovascular, combinado con una exposición más prolongada a FRCV en las mujeres prediabéticas, posiblemente debido a una mayor adiposidad, puede ser la causa del riesgo excesivo de diabetes relacionada con la enfermedad coronaria en las mujeres. Estos mismos autores, en otro estudio de similar naturaleza que el anterior ${ }^{23}$, muestran que el exceso de riesgo de accidente cerebrovascular es mayor en las mujeres, independientemente de las diferencias de sexo en otros FRCV, y sugiere que se debe tener en cuenta que hombres y mujeres experimentan enfermedades relacionadas con la diabetes de manera diferente. Sin duda, los hallazgos de este trabajo coinciden con estos autores, ya que el sexo femenino presentó una mayor incidencia de diabetes, asociada a una mayor presencia de otros FRCV relevantes, determinando un peor perfil de riesgo cardiovascular en comparación con los hombres, así como una tasa más alta de amputación mayor.

En este estudio, además de la diabetes, otros dos FRCV, la hipertensión y la obesidad, presentaron una mayor prevalencia de forma significativa en el sexo femenino.

En el caso de la hipertensión y los FRCV relacionados con el metabolismo sucede algo parecido al diferenciar entre sexos, ya que el porcentaje de mujeres amputadas hipertensas, obesas o que presentan dislipidemia es mayor que el de hombres.

Los estudios publicados afirman que el estado menopáusico y la obesidad asociada es el principal predictor de alteraciones metabólicas de riesgo cardiovascular en las mujeres menopáusicas, observando un incremento en los parámetros antropométricos y en los índices de lípidos plasmáticos en las mujeres posmenopáusicas, estrechamente relacionados con el incremento de la edad ${ }^{24-27}$. Acorde con estos estudios, en los que se concluye que las mujeres sufren un cambio de perfil lipídico a partir de la menopausia, debido fundamentalmente al déficit estrogénico, generando una mayor susceptibilidad para padecer enfermedades cardiovasculares, fueron los resultados de nuestro estudio, en el que hubo una mayor prevalencia de obesidad y dislipidemia en el sexo femenino.

El tabaquismo fue el único FRCV más prevalente en el sexo masculino. Este factor de riesgo es considerado por algunos autores ${ }^{28,29}$ como un FRCV fundamental de enfermedad arterial periférica prevenible. Las amputaciones de MI de pacientes con enfermedad arterial periférica que fuman duplican las de personas que no han fumado nunca. La mayor prevalencia de tabaquismo en los pacientes de sexo masculino de este estudio podría explicar la gran diferencia en el porcentaje de amputaciones realizadas en función del sexo, ya que el porcentaje de hombres amputados fue el triple que el de mujeres.

Considerando que en este estudio el perfil de riesgo cardiovascular de las mujeres fue claramente más desfavorable, salvo para el tabaquismo, cabría esperar que fuese el sexo femenino el que presentase mayor prevalencia de amputaciones de MI, pero solo la tercera parte de los amputados de la muestra son mujeres. 
La mayoría de las mujeres amputadas presentaban una edad avanzada, lo que supone para ellas el cese de la esteroidogénesis ovárica y por tanto de la protección que esta genera en edades más tempranas, favoreciendo la presencia de algunos FRCV modificables como la diabetes, la hipertensión, la dislipidemia y la obesidad. Para algunos autores ${ }^{30,31}$, el incremento de la edad determinaría un aumento en los parámetros antropométricos y en los índices de lípidos plasmáticos en las mujeres posmenopáusicas, convirtiéndose esta variable demográfica, y con ella la menopausia, en la que existe mayor propensión a agrupar FRCV, en factor fundamental en el sexo femenino para el desarrollo de patología vascular periférica.

Este trabajo demostró la asociación entre el sexo femenino, la edad avanzada y tres FRCV (diabetes, hipertensión y obesidad), lo que confirmaría la relación entre el incremento de la edad en el sexo femenino y la presencia de un peor perfil cardiovascular.

Sin embargo, y sin dejar de señalar el importante papel que desempeña la edad en el perfil cardiovascular de riesgo, no podemos afirmar que los resultados de este estudio, con un perfil de riesgo cardiovascular femenino más desfavorable y una prevalencia de amputación mayor más alta en este sexo, sean del todo la causa de una mayor edad en el grupo de las mujeres. La amputación de Ml en las pacientes de este estudio sucedía en peores condiciones vasculares que en los hombres, en edades más avanzadas y con un perfil de riesgo cardiovascular más desfavorable en el momento de la intervención quirúrgica.

La edad podría considerarse como condición necesaria, pero no suficiente, para la ocurrencia y la progresión de la lesión aterosclerótica, causante en la mayoría de las ocasiones de la amputación de MI, puesto que aunque las mujeres amputadas presentaron mayor edad y peor perfil clínico, el sexo masculino fue el más afectado por este tipo de cirugía.

También se relacionó en este estudio el sexo femenino con un nivel superior de amputación de MI, al presentar más frecuencia de amputación mayor que los hombres, con la importancia que tiene el nivel en que se realice este tipo de cirugía para la evolución, el pronóstico y la calidad de vida de los pacientes.

\section{Conclusiones}

Este estudio confirma el importante papel del sexo como factor de riesgo a considerar en la amputación de MI por causa no traumática, ya que el sexo determina un perfil de riesgo cardiovascular más desfavorable en las mujeres con patología vascular y amputadas. También se demostró en las mujeres una asociación negativa con tres FRCV (diabetes, hipertensión y obesidad), así como una mayor predisposición a sufrir una amputación mayor.

Los resultados de este trabajo, junto con la evidencia que se desprende de la práctica clínica y el considerable vacío en el conocimiento del perfil clínico específico de las pacientes amputadas de MI, hacen conveniente el diseño de nuevos estudios multicéntricos, con mayor inclusión de mujeres en la muestra, para establecer definitivamente los parámetros diferenciales en función del sexo del paciente, en los mecanismos biológicos involucrados y su nivel de influencia sobre esta cirugía radical, con preocupante tendencia creciente.

\section{Agradecimientos}

Los autores agradecen al Servicio de Angiología y Cirugía Vascular del Hospital Universitario San Cecilio de Granada, España.

\section{Financiamiento}

La presente investigación no ha recibido ayudas específicas provenientes de agencias del sector público, del sector comercial ni de entidades sin ánimo de lucro.

\section{Conflicto de intereses}

Los autores declaran no tener conflictos de intereses.

\section{Responsabilidades éticas}

Protección de personas y animales. Los autores declaran que para esta investigación no se han realizado experimentos en seres humanos ni en animales.

Confidencialidad de los datos. Los autores declaran que han seguido los protocolos de su centro de trabajo sobre la publicación de datos de pacientes.

Derecho a la privacidad y consentimiento informado. Los autores han obtenido el consentimiento informado de los pacientes y/o sujetos referidos en el artículo. Este documento obra en poder del autor de correspondencia. 


\section{Bibliografía}

1. Kayssi A, Mestral C, Forbes TL, Roche-Nagle G. A Canadian population-based description of the indications for lower-extremity amputations and outcomes. Can J Surg. 2016;59:99-6.

2. Coduras A, Del Llano J, Caiyona M. La diabetes tipo 2 en España: estudio crítico de situación. Madrid: Fundación Gaspar Casal; 2012.

3. Lara Valdivia JE, Otero Enamorado E, Ramos Almeida N. Estudio prospectivo en pacientes amputados de miembros inferiores. Racionalización en el seguimiento. Rev Cubana Ortop Traumatol. 2001;15:46-50.

4. Rubio JA, Salido C, Albarracín A, Jiménez S, Álvarez J. Incidencia de amputaciones de extremidades inferiores en el área 3 de Madrid. Estudio retrospectivo del periodo 2001-2006. Rev Clin Esp. 2010;210:57-100.

5. Calle-Pascual AL, García-Torre N, Moraga I, Díaz JA, Durán A, Moñux G. Epidemiology of nontraumatic lower-extremity amputation in area 7, Madrid, between 1989 and 1999: a population-based study. Diabetes Care. 2001:24:1686-9.

6. Almaraz MC, Soriguer F, Zamorano D, Ruiz de Adara S, González E, Esteva I, et al. Incidencia de amputaciones de extremidades inferiores en la población con diabetes mellitus de Málaga (1996-1997). Aten Primaria. 2000;26:677-80.

7. The Global LEA Study Group. Epidemiology of lower extremity amputation in centres in Europe, North America and East Asia. $\mathrm{Br} J$ Surg 2000;87:328-7

8. Ardila Merchán F, Ros Díe E. Amputaciones de las extremidades inferiores en patología vascular. Recomendaciones para el diagnóstico y tratamiento en angiología y cirugía vascular. Sevilla: Servicio Andaluz de Salud; 2006. p. 51-6.

9. Davie-Smith F, Paul L, Nicholls N, Stuart WP, Kennon B. The impact of gender, level of amputation and diabetes on prosthetic fit rates following major lower extremity amputation. Prosthet Orthot Int. 2017;41:19-25.

10. Molino AM, Fernández-Cruz A, Patiño R. Amputación no traumática de miembros inferiores en pacientes de la comunidad de Madrid 1997-2005: epidemiología y estimación de los costes hospitalarios. Madrid: Universidad Complutense de Madrid; 2007.

11. Claver García L. Riesgo cardiovascular en la mujer. Universidad Pública de Navarra. 2014. (Consultado el 6 de septiembre de 2019.) Disponible en: http:/academicae unavarra.es/bitstream/handle/2454/11232/LauraClaverGarcia.pdf?sequence $=1$

12. Muniesa JM, Pou M, Marco E, Boza R, Guillén A, Duarte E, et al. Calidad de vida en pacientes con amputación de extremidad inferior. Rehabilitación. 2009:43:28-3.

13. Sereday M, Damiano M, Lapertosa S, Cagide A, Bragagnolo JC. Amputaciones de miembros inferiores en diabéticos y no diabéticos en el ámbito hospitalario. Revista de la Asociación Latinoamericana de Diabetes - ALAD. 2009;17:9-15.

14. Henríquez L. Calidad de vida de los pacientes amputados de la extremidad inferior. Rev Med Cos Cen. 2009:589:267-3.

15. Farro L, Tapia R, Bautista L, Montalvo R, Iriarte H. Características clínicas y demográficas del paciente amputado. Rev Med Hered. 2012;23:240-3.
16. Cabrera Zamora JL, Hernández Seara A, Viña Cisnero H, Hondares Guzmán MC, López Díaz M, Licor García E, et al. Características de las amputaciones mayores en pacientes con isquemia arterial aguda trombótica de miembros inferiores. Rev Cubana Angiol Cir Vasc. 2013;14(1).

17. Lara R, Lozano M. Isquemia crítica de miembros inferiores: una enfermedad cada vez más prevalente. Med Clin. 2011;136:106-8.

18. Mostaza JM, Puras E, Álvarez J, Cairols M, García-Rospide V. Características clínicas y evolución intrahospitalaria de los pacientes con isquemia crítica de miembros inferiores: estudio ICEBERG. Med Clin. 2011;136:91-138.

19. Hoffmann F, lacks A. Diabetes 'epidemic' in Germany? A critical look at health insurance data sources. Exp Clin Endocrinol Diabetes. 2012;120:410-5.

20. Del Valle M. Epidemiología de la diabetes. XIX Congreso Latinoamericano de Patología Clínica/ML. La Habana: ALAPAC; 2009. (Consultado el 12 de enero de 2020.) Disponible en: http://www.sld.cu/galerias/pdf/uvs/ patologiaclinica/epidemiologia de la diabetes1-2009.pdf

21. Peek ME. Gender differences in diabetes related lower extremity amputations. Clin Orthop Relat Res. 2011;469:1951-5.

22. Peters SAE, Huxley R, Woodward M. Diabetes as risk factor for incident coronary heart disease in women compared with men: a systematic review and meta-analysis of 64 cohorts including 858,507 individuals and 28,203 coronary events. Diabetologia. 2014;57:1542-51.

23. Peters SAE, Huxley RR, Woodward M. Diabetes as a risk factor fo stroke in women compared with men: a systematic review and meta-analysis of 64 cohorts, including 775,385 individuals and 12,539 strokes. Lancet. 2014;383:1973-80.

24. Dasgupta S, Salman M, Lokesh S, Xaviour D, Saheb SY, Prasad BV et al. Menopause versus aging: the predictor of obesity and metabolic aberrations among menopausal women of Karnataka, South India. J Midlife Health. 2012;3:24-30.

25. Zylbersztejn HM, Kusznier SG, Olivares GB, Oviedo GA, Kanterewicz LG, Elizalde RJ. Epidemiología de los factores de riesgo vascular en mujeres climatéricas. Experiencia de un consultorio multidisciplinario de climaterio en un hospital público de Buenos Aires. Rev Argen Cardiol. 2013:81:336-3.

26. Matthews KA, Crawford SL, Chae CU, Everson-Rose SA, Sowers MF, Sternfeld $\mathrm{B}$, et al. Are changes in cardiovascular disease risk factors in midlife women due to chronological aging or to the menopausal transition? Results from the Study of Women's Health Across the Nation (SWAN). J Am Coll Cardiol. 2009;54:2366-3.

27. Feliciano T. Menopausia y riesgo cardiovascular. Rev Centroam Obstet Ginecol. 2011;16:34-6.

28. Lekuona Goya I, Salcedo Arruti A, Morillas Bueno M, Umaran Sánchez J. Tabaco y enfermedad arterial no coronaria. Intervenciones para el abandono del hábito tabáquico. Rev Esp Cardiol. 2009:9:39-48.

29. Burke A, FitzGerald GA. Oxidative stress and smoking-induced vascular injury. Prog Cardiovasc Dis. 2003;46:79-90.

30. Lejsková M, Alušík S, Valenta Z, Adámková S, Pit'ha J. Natural postmenopause is associated with an increase in combined cardiovascular risk factors. Physiol Res. 2012;61:587-96.

31. Colpani V, Oppermann K, Spritzer PM. Association between habitual physical activity and lower cardiovascular risk in premenopausal, perimenopausal, and postmenopausal women: a population-based study. Menopause. 2013;20:525-1. 